\title{
PROPRIEDADES FÍSICO-QUÍMICAS E DE ESTABILIDADE DE ÓLEO DE COCO OBTIDOS POR PROCESSOS ARTESANAIS E INDUSTRIAIS
}

\author{
M.M. B. OLIVEIRA ${ }^{1}$, T.H.C. de MELO $^{2}$,S.C. de MELO FILHO ${ }^{3}$ \\ 1,2 Universidade Federal de Pernambuco, Centro de Ciências da Saúde, Departamento de \\ Ciências Farmacêuticas \\ ${ }^{3}$ Universidade Federal de Pernambuco, Centro de Ciências da Saúde, Departamento de \\ Nutrição
}

E-mail para contato: mmbonutricao@gmail.com

\begin{abstract}
RESUMO - O óleo de coco vem obtendo um grande destaque pelos meios de comunicação quando se fala no seu potencial terapêutico, mas dados relativos a estas propriedades na literatura ainda são escassos e ainda controversos. Caracterizar as propriedades físico-químicas e de estabilidade do óleo de coco obtidos por processos artesanais e industriais. Consistiu na reprodução de técnicas de obtenção de amostras de óleo de coco artesanal com e sem fermentação do extrato aquoso da polpa do coco, submetidos ou não a aquecimento final e amostras de óleo industrializado. Submeteramse todas as amostras a caracterização físico-química através de analise de $\mathrm{pH}$, densidade, Índice de refração (IR), e testes de estabilidade química (índice de acidez, acidez em ácido Oleico, acidez em acido Láurico, índice de peróxido e teste de Kreis). Os resultados obtidos de densidade, $\mathrm{pH}$, IR, e densidade demonstram que os óleos de coco tanto artesanais quanto obtidos por tanto processos industriais quanto por processos artesanais possuem características fisico-químicas adequadas indicativo para a estabilidade química frente a processos oxidativos. Os índices de acidez e índices de peroxido encontrados estavam de acordo com o padrão estabelecido pela Resolução $N$ 270, de 22 de Setembro de 2005, da Agência Nacional da Vigilância Sanitária - ANVISA. A metodologia empregada na extração artesanal não influenciou nas propriedades fisico-químicas, pois esses parâmetros encontraram-se dentro os limites previstos pela legislação vigente para óleos vegetais e são semelhantes as amostras industrializadas.
\end{abstract}

Palavras chave: Óleo de coco. Caracterização físico-química. Estabilidade. Processo Artesanal.

ABSTRACT - Coconut oil has been gaining prominence in the media when discussing its therapeutic potential, but data on these properties in the literature are still scarce and still controversial. This work proposes to characterize as physical-chemical and stability properties of coconut oil obtained by industrial and industrial processes. Consistency in the reproduction of techniques for obtaining coconut oil samples, artisanal and without fermentation of aqueous extract of the coconut pulp, submitted or not to final temperature and industrialized oil samples. All samples were subjected to physical-chemical characterization by analysis of $\mathrm{pH}$, density, refractive index (IR), moisture content and ash; And tests of chemical stability (acidity index, acidity in acrylic, acidity in lactic acid, peroxide index and Kreis test). The results of density, $p H$, IR, density, moisture and total 
ash show that coconut glasses, both handcrafted and obtained by industrial processes, have adequate chemical physical characteristics indicative of chemical stability against oxidative processes. The acid indexes and sodium emission indices in series are in conformity with the standard established by Resolution N 270, of September 22, 2005, of the National Agency of Sanitary Surveillance - ANVISA. The methodology used in the artisanal extraction did not influence the physical lodging, chemical, poets such as the limits established for the current legislation for vegetable oils are like industrialized samples.

Keywords: Coconut oil. Physical-chemical characterization. Stability. Artisanal process.

\section{INTRODUÇÃO}

Uma das fontes de gordura vegetal recentemente veiculada ao preparo culinário e também introduzido na elaboração de produtos alimentícios é o óleo de coco, amplamente utilizado na culinária em diversos países e também empregado na produção de cosméticos, lubrificantes, tintas, fármacos, biodiesel, entre outros (MARINA et al., 2009).

O óleo de coco é obtido a partir da polpa do coco fresco maduro (Cocos nucifera L.), com composição de $70 \%$ de gorduras saturadas e destes, $80 \%$ dos ácidos graxos são de cadeia média (AGCM), o restante é composto ácidos graxos insaturados, sendo encontrado na forma líquida (DEBMANDAL et al., 2011). Dos ácidos graxos saturados presentes os principais são o capróico, caprílico, cáprico, láurico, mirístico, palmítico e esteárico; e dos insaturados, os ácidos oleico e linoleico. Destes, $47 \%$ é do ácido láurico que se encontra em maior quantidade. As gorduras láuricas são utilizadas na indústria cosmética e alimentícia, dadas as suas propriedades físicas e resistência à oxidação, são muito empregadas na preparação de gorduras especiais para confeitaria, sorvetes, margarinas e substitutos de manteiga de cacau (LAWSON, 1999).

Quando aquecido o óleo de coco virgem não perde suas caraterísticas nutricionais, sendo assim apontado como um óleo estável. Do ponto de vista nutricional é considerado saudável por não apresentar gordura trans que é gerada no processo hidrogenação, presentes em outros óleos de origem vegetal como os de soja, milho, canola entre outros (MACHADO et al., 2006).

O óleo de coco vem sendo utilizado como coadjuvante do tratamento da obesidade pelo alto teor de ácidos graxos de cadeira média, sendo que esses lipídeos são facilmente oxidados e, geralmente, não são armazenados no tecido adiposo, fazendo com que a taxa do metabolismo basal seja diminuída (LIAU et al., 2011).

Neste sentido, este trabalho buscou avaliar as propriedades físico-químicas e de estabilidade de amostras de óleo de coco artesanal e industrial, de forma comparativa com a legislação vigente.

\section{MATERIAIS E MÉTODOS}

\subsection{MATERIAL DE ESTUDO}

O material de estudo foi composto por óleos de coco produzidos artesanalmente conforme metodologias de extração mais encontradas segundo pesquisa em artigos de bases de dados, sites e páginas de vídeos, e de óleos de coco obtidos industrialmente. Foram identificados 6 métodos de obtenção artesanal mais frequentes: prensagem a frio 
da polpa do coco, centrifugação do extrato do coco a frio, fermentação do extrato do coco com e sem aquecimento final do sobrenadante, separação de fases do extrato aquoso de coco sem e com aquecimento final do sobrenadante. Foram selecionados os métodos de fermentação e sedimentação com separação de fases, em ambos os casos com aquecimento final para extração do óleo e sem aquecimento final, totalizando 4 formas diferentes de obtenção do óleo a partir de extrato aquoso (leite de coco).

A matéria-prima utilizada para obtenção do óleo de coco foram coco natural para elaboração do óleo de coco artesanal e as amostras dos óleos de coco industrializados foram obtidas em farmácias comerciais e supermercados da cidade do Recife - PE. As amostras de óleo de coco industrializado foram codificadas como AI 1 (amostra industrializada 1), AI 2 (amostra industrializada 2), AI 3 (amostra industrializada 3 ) e AI 4 (amostra industrializada 4).

Utilizou-se aproximadamente $400 \mathrm{~g}$ de coco ralado para obtenção de cada tipo de processamento de óleo artesanal. Seguiu-se inicialmente com a extração do "leite de coco" que é o extrato aquoso da polpa do coco, obtido em processador com adição do coco ralado com $800 \mathrm{~mL}$ de água potável, sendo processado por aproximadamente 1 minuto. A mistura obtida foi filtrada em pano de algodão e o extrato separado da polpa que foi condicionado em recipiente com tampa. Este processo foi realizado para a obtenção dos 04 tipos de extração de óleo de coco artesanais. As amostras artesanais foram codificadas como AC 1 (amostra artesanal 1), AC 2 (amostra artesanal 2), AC 3 (amostra artesanal 3) e AC 4 (amostra artesanal 4).

Foram atribuídas quatro métodos para obtenção de óleo de coco artesanal: Procedimento 01. Extração por fermentação natural (48h) com separação do óleo de coco com aquecimento final - Amostra AC 1; Procedimento 02. Extração por fermentação natural (48h) com separação do óleo de coco sem aquecimento - Amostra AC 2; Procedimento 03. Extração do óleo de coco sem fermentação (separação de fase em repouso de 48h sob refrigeração) com aquecimento final - Amostra AC 3; e Procedimento 04. Extração do óleo de coco sem fermentação (separação de fase em repouso de $48 \mathrm{~h}$ sob refrigeração) sem aquecimento final - Amostra AC 4.

Para os procedimentos das amostras AC1 e AC2 que utilizaram fermentação natural (microbiota da flora), foram mantidos dois recipientes plásticos fechados contendo os extratos de coco em temperatura ambiente por $48 \mathrm{~h}$. Após as $48 \mathrm{~h}$, foram levados a refrigeração $\left( \pm 8^{\circ} \mathrm{C}\right)$ por $6 \mathrm{~h}$, para a solidificação do sobrenadante obtido pela fermentação. Posteriormente, a amostra AC1 foi levada a aquecimento até obter-se a separação do óleo da polpa fermentada e a AC2 permaneceu em temperatura ambiente para obter-se a separação de fases. Para os outros dois procedimentos de extração das amostras AC3 e AC4, os recipientes contendo os extratos foram acondicionados sob refrigeração $\left( \pm 8^{\circ} \mathrm{C}\right)$ por $48 \mathrm{~h}$, para separação de fases e obtenção de sobrenadante. Após esse período, a amostra AC3 recebeu aquecimento para obtenção do óleo e a outra permaneceu em temperatura ambiente para retirada do sobrenadante e separação do óleo da polpa (AC 4).

\subsection{CARACTERIZAÇÃO FÍSICO - QUÍMICA DOS ÓLEOS DE COCO}

As propriedades físico-químicas das amostras artesanais e industrializadas foram determinadas em triplicata de acordo com Santos et al., (2006) e Santos (2007) com modificações e segundo os procedimentos analíticos descritos pelo Instituto Adolfo Lutz - IAL (2008). 


\section{Determinação de pH e índice de acidez}

$\mathrm{O} \mathrm{pH}$ foi determinado pelo método potenciométrico. Foi pesado aproximadamente $10 \mathrm{~g}$ de óleo de coco em temperatura ambiente. $\mathrm{O} \mathrm{pH}$ foi medido por meio da inserção dos eletrodos na amostra após agitação e repouso, realizado em triplicata (BRASIL, 2008).

Para determinar o índice de acidez, em um erlenmeyer de $125 \mathrm{~mL}$ previamente tarado, foi adicionado 2,0g de óleo de coco. Em seguida foi adicionado à mistura de 16,6 $\mathrm{ml}$ de éter etílico e $8,3 \mathrm{ml}$ de álcool etílico $(2: 1) \mathrm{v} / \mathrm{v}$ e agitou-se até dissolução da amostra. Foram adicionadas 2 gotas de fenolftaleína e a mistura foi titulada com solução padronizada de $\mathrm{NaOH} 0,1 \mathrm{M}$, até o aparecimento de uma coloração levemente rósea (BRASIL, 2008). $\mathrm{O}$ índice de acidez foi calculado segundo: Índice de acidez $=\mathrm{V}$ x f $\mathrm{x}$ 5,61P . Em que: $\mathrm{V}=$ volume da solução de $\mathrm{NaOH}$ gasto na titulação em $\mathrm{mL}$; $\mathrm{f}=$ fator de correção da solução de $\mathrm{NaOH} ; \mathrm{P}=$ número de gramas da amostra. Para expressar o índice de acidez como acidez em ácido oléico, o resultado foi dividido por 1,99. Para expressar o índice de acidez em ácido láurico foi usada a equação: Índice de acidez (\% ácido láurico) $=\mathrm{V}$ x N x 200/P. Em que: V= volume da solução de $\mathrm{NaOH}$ gasto na titulação em $\mathrm{mL} ; \mathrm{N}$ $=$ Normalidade da solução de $\mathrm{NaOH}$ padronizada; $200=$ fator de gramas para ácido láurico; $\mathrm{P}=$ número de gramas da amostra.

\section{Densidade e índice de refração}

Em uma proveta de $25 \mathrm{ml}$ previamente tarada, foram adicionados $10 \mathrm{ml}$ de óleo pesados em uma balança analítica, registrando-se o peso obtido. Para a determinação da densidade das amostras de óleo de coco foi usada a equação a seguir: $\mathrm{D}=$ Peso da amostra (g)/ Volume da amostra (mL).

Para determinação do índice de refração foi utilizado o refratômetro modelo ausJENA 375320. Após limpeza dos prismas do aparelho com algodão e éter etílico cada amostra foi levada a banho-maria para atingir a temperatura de $40^{\circ} \mathrm{C}$. Em seguida, algumas gotas de óleo foram colocadas no prisma inferior, os prismas foram fechados. Com ajuste do instrumento e da luz a leitura foi realizada e o índice de refração determinado (BRASIL, 2008).

\section{Determinação do índice de peróxidos}

Para a determinação do índice de peróxidos, em um Erlenmeyer de $125 \mathrm{~mL}$ previamente tarado, foi colocado $5 \mathrm{~g}$ de óleo de coco. Em seguida foi adicionado $30 \mathrm{~mL}$ de solução de ácido acético-clorofórmio (3:2) v/v e agitou-se até dissolução da amostra. Foi adicionado $1 \mathrm{~g}$ de Iodeto de Potássio e $30 \mathrm{~mL}$ de água destilada e homogeneizou-se até completa dissolução, seguiu-se a titulação com solução padronizada de $\mathrm{Na}_{2} \mathrm{~S}_{2} \mathrm{O}_{3}$ até que a cor amarela desaparecesse completamente (BRASIL, 2008). O índice de peróxidos foi calculado segundo a equação: I.P. $=(\mathrm{Vb}-\mathrm{Va}) \times \mathrm{C} \times \mathrm{F} \times 1000 / \mathrm{m}$. Em que: I.P. $=$ índice de peróxido $(\mathrm{mEq}$ de peróxido/1.000 $\mathrm{g}$ de amostra); $\mathrm{Va}=$ volume $(\mathrm{mL})$ da solução de $\mathrm{Na}_{2} \mathrm{~S}_{2} \mathrm{O}_{3} 0,01 \mathrm{~mol} / \mathrm{L}$ gasto na titulação da amostra; $\mathrm{Vb}=$ volume $(\mathrm{mL})$ da solução de $\mathrm{Na}_{2} \mathrm{~S}_{2} \mathrm{O}_{3} 0,01 \mathrm{~mol} / \mathrm{L}$ gasto na titulação da prova em branco; $\mathrm{C}=$ concentração molar da solução de $\mathrm{Na}_{2} \mathrm{~S}_{2} \mathrm{O}_{3}$ igual a $0,01 \mathrm{~mol} / \mathrm{L} ; \mathrm{F}=$ fator de correção da solução de $\mathrm{Na}_{2} \mathrm{~S}_{2} \mathrm{O}_{3} 0,01$ $\mathrm{mol} / \mathrm{L} ; \mathrm{m}=$ massa da amostra $(\mathrm{g})$.

\section{Teste de Kreis}

Em um tubo de ensaio foi adicionado $5 \mathrm{~mL}$ de óleo de coco e $5 \mathrm{~mL} \mathrm{HCl}$, agitando-se por 30 segundos. Em seguida, foi adicionado $5 \mathrm{~mL}$ de floroglucina $0,1 \% \mathrm{em}$ éter, agitando-se novamente por 30 segundos e o tubo foi deixado em repouso por 10 minutos (BRASIL, 2008). 


\section{RESULTADOS E DISCUSSÃO}

Com relação às técnicas de extração artesanais, tanto o procedimento 02 (AC2), usando fermentação, quanto o procedimento 04 (AC4) sem fermentação, em que em ambas a extração final caracterizava-se por ser a frio, ou seja sem aquecimento do sobrenadante final, não foi possivel a reprodução das técnicas de extração. Os procedimentos em que extração final do óleo de coco foi a quente, ou seja, com utilização de aquecimento do extrato fermentado ou não (AC1 e $\mathrm{AC} 3$ respectivamente), foi possível obter as amostras em quantidades satisfatórias.

A densidade determina a quantidade de matéria que está presente em uma unidade de volume. A forma como as cadeias se atraem e sua capacidade de se empacotar determinam várias propriedades físico-químicas entre elas à densidade. As densidades de óleos tendem ser $0,910-0,930 \mathrm{~g} / \mathrm{mL}$ em temperatura ambiente, tendendo a diminuir com o aumento da temperatura. (FANNEMA, 2007) De acordo com Codex Alimentarius (2003) a densidade aparente para óleo de coco é de $0,890-0,921 \mathrm{~g} / \mathrm{mL}$ sendo assim todas as amostras encontram-se abaixo do limite estabelecido (Tabela 1). Sugere-se que essas pequenas variações sejam reflexo das condições de processamento e armazenamento (RIBEIRO, 2007).

Tabela 1 - Densidade, pH e Índice de Refração (IR) de óleos de coco obtidos por processos artesanais e industriais.

\begin{tabular}{cccc}
\hline AMOSTRAS & $\begin{array}{c}\text { Densidade }(\mathbf{g} / \mathbf{m L}) \\
(\mathbf{X} \pm \mathbf{D P})\end{array}$ & $\begin{array}{c}\mathbf{p H} \\
(\mathbf{X} \pm \mathbf{D P})\end{array}$ & $\mathbf{I R}$ \\
\hline AC 1 & $0,859( \pm 0,018)$ & $5,21( \pm 0,304)$ & 1,455 \\
AC 3 & $0,852( \pm 0,028)$ & $5,51( \pm 0,721)$ & 1,456 \\
AI 1 & $0,830( \pm 0,028)$ & $4,96( \pm 0,066)$ & 1,455 \\
AI 2 & $0,825( \pm 0,028)$ & $4,73( \pm 0,381)$ & 1,455 \\
AI 3 & $0,854( \pm 0,028)$ & $4,85( \pm 0,212)$ & 1,455 \\
AI 4 & $0,841( \pm 0,028)$ & $5,36( \pm 0,509)$ & 1,455 \\
\hline
\end{tabular}

Legenda: $\mathrm{X}=$ média; $\mathrm{DP}=$ desvio padrão.

A avaliação do $\mathrm{pH}$ das amostras, é de extrema importância para determinar se o óleo é de boa qualidade, se não sofreu nenhum processo de degradação no momento da extração. No presente estudo as amostras de óleo apresentaram uma faixa de $\mathrm{pH}$ alta quando comparada com o estudo de Santos (2013), que encontrou para óleo de coco artesanal o valor de 3,33 e para o industrial 3,50. Essa discrepância poderia ser atribuída a várias diferenças como o cultivo do coco para extração e armazenamento.

Para os índices de refração, quando tido por base o Codex Alimentarius (2003) que determina que os valores de refração do óleo a $40^{\circ} \mathrm{C}$ devem estar na faixa de 1.448 1.450 para que óleo de coco seja de boa qualidade, os valores obtidos nos experimentos aproximam-se aos valores estabelecidos na legislação, o que indica boa qualidade dos óleos avaliados (Tabela 1).

Com relação aos parâmetros relativos a estabilidade química dos óleos de coco foram avaliados os indices de acidez, com acidez em ácido oleico e em acido laurico já que este é o acido graxo majoritario, que são apresentados na Tabela 2. 
Tabela2 -Índice de Acidez (mg de $\mathrm{NaOH} / \mathrm{g}$ de óleo), Acidez em ácido óleico das amostras (\%) e Acidez em ácido láurico das amostras (\%) de óleos de coco artesanais e industrializados.

\begin{tabular}{cccc}
\hline AMOSTRAS & $\begin{array}{c}\text { Índice de acidez } \\
(\mathbf{X} \pm \mathbf{D P})\end{array}$ & $\begin{array}{c}\text { Acidez em ac. Oleico } \\
\mathbf{( \% )} \\
(\mathbf{X} \pm \mathbf{D P})\end{array}$ & $\begin{array}{c}\text { Acidez em ac. } \\
\text { Láurico (\%) } \\
(\mathbf{X} \pm \mathbf{D P})\end{array}$ \\
\hline AC 1 & $1,066( \pm 0,093)$ & $0,527( \pm 0,059)$ & $3,833( \pm 0,429)$ \\
AC 3 & $0,954( \pm 0,449)$ & $0,411( \pm 0,130)$ & $2,991( \pm 0,942)$ \\
AI 1 & $1,291( \pm 0,016)$ & $0,646( \pm 0,011)$ & $4,070( \pm 0,777)$ \\
AI 2 & $0,937( \pm 0,007)$ & $0,558( \pm 0,119)$ & $4,063( \pm 0,821)$ \\
AI 3 & $0,773( \pm 0,224)$ & $0,388( \pm 0,112)$ & $2,828( \pm 0,821)$ \\
AI 4 & $0,775( \pm 0,236)$ & $0,389( \pm 0,119)$ & $2,835( \pm 0,866)$ \\
\hline
\end{tabular}

Legenda: $\mathrm{X}=$ média; $\mathrm{DP}=$ desvio padrão.

A determinação da acidez é um parâmetro importante para os óleos para avaliar o seu estado de conservação. Um elevado índice de acidez indica que o óleo ou gordura está sofrendo quebras em sua cadeia, liberando seus constituintes principais: os ácidos graxos. E é por esse motivo que o cálculo desse índice é de extrema importancia importância na avaliação do estado de deterioração (rancidez hidrolítica ou oxidativa) do óleo. O índice de acidez é definido como o número de $\mathrm{mg}$ de hidróxido de potássio necessário para neutralizar os ácidos livres em um grama da amostra (COSTA et al., 2006). Os valores de acidez determinados nas amostras encontram-se abaixo do valor estabelecido para óleos brutos (4,0 mg NaOH/g de óleo) segundo a RDC 270/2005 ANVISA que regulamenta óleos vegetais, gorduras vegetais e creme vegetal e diz que os requisitos específicos são Acidez e Índice de Peróxido (BRASIL, 2005).

$\mathrm{Na}$ literatura não foi encontrado o valor padrão para a classificação do óleo de coco como virgem ou extra virgem, por isso foi utilizada como parametro a RDC $270 / 2005$ e os valores atribuidos para o azeite de oliva uma vez que ambos são classificados como óleos brutos. Os óleos industrializados são considerados extra virgem quando seu indice de acidez é inferior a $0,5 \%$ em ácido óleico, assim só as amostras industrializadas 2, 3 e 4 podem ser classificadas assim. Em todas as amostras industrializadas foram encontrados em seus rotulos a designização extravirgem, porém a amostra AI encontra-se em desacordo com a legislação vigente pois apresenta resultado superior ao preconizado.

Os resultados de índice de acidez (\%) de ácidos graxos livres expressos em ácido láurico apresentaram-se menores que a recomendação de $2,5 \%$ valor máximo recomendado por Masson (1999) para óleos vegetais (Tabela 3). No Brasil, as principais fontes de ácido láurico são os óleos de coco, de palmiste e de babaçu (COSTA, 2006). Por esse motivo a acidez em \% de ácido láurico foi avaliada obtendo valores dentro do limite $(<0,3 \%)$ quando comparadas com o Codex Alimentarius (2003). Deste modo, os índices de acidez apresentaram resultados positivos, mostrando o bom estado de conservação dos óleos e que mesmo submetido a temperaturas altas durante a extração para os óleos artesanais, pode-se afirmar que esta condição não interferiu na estabilidade química a ponto de deteriorar as amostras. 
O teste de Kreis é um método rápido colorimétrico qualitativo que serve para a indicação da ocorrência de oxidação lipídica, no inicio do desenvolvimento do ranço, em que para teste positivo observa - se a coloração rósea avermelhada (HAMILTON, 1983). Todas as amostras desse estudo foram negativas para o teste (Tabela 3).

Tabela 3- Teste de Kreis e Indice de Peróxidos (meq/Kg ) de óleos de coco artesanais e industrializados

\begin{tabular}{c|c|c}
\hline AMOSTRAS & Teste de Kreis & $\begin{array}{c}\text { Índice de Peroxido } \\
(\mathbf{X} \pm \mathbf{D P})\end{array}$ \\
\hline AC 1 & Negativo & $2,337( \pm 0,0494)$ \\
\hline AC 3 & Negativo & $3,546( \pm 1,759)$ \\
\hline AI 1 & Negativo & $2,419( \pm 0,0827)$ \\
\hline AI 2 & Negativo & $2,436( \pm 0,1379)$ \\
\hline AI 3 & Negativo & $2,365( \pm 0,0749)$ \\
\hline AI 4 & Negativo & $3,578( \pm 1,682)$ \\
\hline
\end{tabular}

Legenda: $\mathrm{AC} 1=$ amostra artesanal $1 ; \mathrm{AC} 3=$ amostra artesal 3 ; $\mathrm{AI} 1=$ amostra industrializada $1 ; \mathrm{AI} 2=$ amostra industrializada 2; AI $3=$ amostra industrializada 3; AI 4= amostra industrializada 4.; X = média; $\mathrm{DP}=$ desvio padrão.

A determinação do índice de peróxido possibilita saber o grau de degradação da matéria-prima, que segundo Poyato et al. (2014) decorre da oxidação dos ácidos graxos insaturados, formando hidroperóxidos que não possuem cheiro nem gosto característicos que pode já ocorrer no momento da extração, podendo também afetar as suas qualidades nutricionais. A RDC 270/2005 da ANVISA (Brasil, 2005) diz que um óleo para ser considerado bom para consumo precisa ter um valor máximo de $10 \mathrm{meq} / \mathrm{Kg}$ de óleo ou gordura. O índice de peróxidos das amostras encontra-se dentro do limite estabelecido pela legislação (Tabela 3).

\section{CONCLUSÃO}

As metodologias empregadas na extração artesanal não influenciaram nas propriedades físico-químicas, visto que esses parâmetros encontraram-se dentro os limites previstos pela legislação vigente para óleos vegetais e são semelhantes as amostras industrializadas. Estudos complementares devem ser realizados sobre o processamento deste óleo bem como a estabilidade térmica frente a utilização em aquecimento prolongado, pois na literatura ainda são escassos resultados e parâmetros.

\section{REFERÊNCIAS}

BRASIL. Ministério da Saúde: Agência Nacional de Vigilância Sanitária (ANVISA). 2005. Resolução RDC $n^{\circ}$ 470, de 22 de setembro de 2005. Aprova o Regulamento Técnico: "regulamento técnico para óleos vegetais, gorduras vegetais e creme vegetal".

BRASIL. INSTITUTO ADOLFO LUTZ - Normas Analíticas; métodos químicos e físicos para a análise de alimentos. 4 ed. São Paulo: Instituto Adolfo Lutz, 2008.

CODEX ALIMENTARIUS. Codex Standard for olive oils, and olive pomace oils, Roma, FAO/WHO, 1981. Codex Stan 33 (revisão 2-2003). 
DEBMANDAL, M.; MANDAL, S. Coconut (Cocos nucifera L.: Arecaceae): In health promotion and disease prevention. Asian Pacific Journal of Tropical Medicine. 2011.

FENNEMA, O. R.; DAMODARAN, S.; PARKIN, K. L. Química de Alimentos de Fennema $4^{\text {a }}$ ed. - Editora Artmed. 2010.

HAMILTON, R. J.; ROSSELL, J. B.; HUDSON, B. J. F.; LÖLIGER, J. In Rancidity in Foods Applied Science Publishers LTD. p. 1. 1983.

LAWSON, H. Aceites y grasas alimentarios: tecnología, utilización y nutrición. Acribia, 1999.

LIAU, K. M.; LEE, Y. Y.; CHEN, C. K.; RASOOL, A. H. G. An open-label pilot study to assess the efficacy and safety of virgin coconut oil in reducing visceral adiposity. ISRN Pharmacology, 2011.

MACHADO, G. C.; CHAVES, J. B. P.; ANTONIASSI, R. Composição em ácidos graxos e caracterização física e química de óleos hidrogenados de coco babaçu. Revista Ceres, v.53, n. 308, p. 463, 2006.

MARINA A.M.; CHE MAN Y.B.; AMI I. Chemical properties of virgin coconut oil. J Am Oil Chemists Soc. v.86, n.30, p.301-307, 2009.

MASSON, L. et. al. Fat deterioration in deep fat frying french fries potatoes at restaurant and food shop sector. Grasas y Aceites, v.50, n.6, p.460-468, 1999.

POYATO, C.; ANSORENA, D.; NAVARRO-BLASCO, I.; ASTIASARÁN, I. A novel approach to monitor the oxidation process of different types of heated oils by using chemometric tools. Food Research International, v.57, p.152-161, 2014.

RIBEIRO, E. P. Química de alimentos. 2 ed. São Paulo: Blucher p.125, 2007.

SANTOS, J. C. O. Thermal Characterization of the Favelone Oil (Cnidoscolus phyllacanthus). Journal of Food Technology. v. 5, p. 77-78, 2007.

SANTOS J. R. M., MARTINS J. S., FREIRE M. S., NETO N. S., et al., Caracterizaçao físico-química do oleo de coco obtido artesanalmente. Congresso Norte Nordeste de Química. 8-12 April, Natal (Brasil) 2007; http://annq.org/eventos/ upload/1362501330.pdf 